\title{
« L'art a le pouvoir de l'indéfinissable ». Entretien avec Banu Cennetoglu
}

"Art has the power of its undefinability". An interview with Banu Cennetoglu

\section{Antonia GARCIA CASTRO et Miriam Perier}

\section{(2) OpenEdition}

12 Journals

\section{Édition électronique}

URL : https://journals.openedition.org/conflits/1998

DOI : 10.4000/conflits. 1998

ISSN : $1777-5345$

Éditeur :

CECLS - Centre d'études sur les conflits - Liberté et sécurité, L'Harmattan

\section{Édition imprimée}

Date de publication : 1 décembre 2005

ISBN : 2-296-00230-7

ISSN : 1157-996X

\section{Référence électronique}

Antonia GARCIA CASTRO et Miriam Perier, « «L'art a le pouvoir de l'indéfinissable ». Entretien avec Banu Cennetoglu », Cultures \& Conflits [En ligne], 60 I hiver 2005, mis en ligne le 24 mars 2006, consulté le 27 septembre 2021. URL : http://journals.openedition.org/conflits/1998 ; DOI : https:// doi.org/10.4000/conflits. 1998

Ce document a été généré automatiquement le 27 septembre 2021.

Creative Commons License 


\title{
«L'art a le pouvoir de l'indéfinissable ». Entretien avec Banu Cennetoglu
}

\author{
"Art has the power of its undefinability". An interview with Banu Cennetoglu
}

Antonia GARCIA CASTRO et Miriam Perier

1 Ce nouveau dossier de la rubrique « Regards sur l'entre-deux » porte son attention sur deux travaux de l'artiste Banu Cennetoglu, respectivement intitulés « False Witness » et « The List ».

2 La première a comme point de départ une visite au centre de demandeurs d'asile Ter Apel (Pays-Bas). La seconde se présente comme une liste de 6336 réfugiés morts, depuis 1993, au sein des frontières de l'Europe, liste de noms que l'artiste entend diffuser dans la ville d'Amsterdam. CEuvre? Dispositif? Qu'en est-il du processus de création lorsque le regard de l'artiste se porte sur les réalités les plus crues ? C'est à la démarche de cette artiste $d u$ visuel que nous nous sommes tout particulièrement intéressées et à l'originalité non seulement de son regard mais aussi et surtout de sa position accordant une place centrale à la médiation et à l'avènement d'un dialogue possible entre artiste et public. A cet égard "questionnements » semble être le mot clé. Questionnements motivant l'artiste, son envie de faire et ses façons de faire. Questionnements que l'on entend soumettre au tiers observateur confronté à une œuvre. Si trait d'union il y a pour Banu Cennetoglu entre les mots art et politique il est fourni par la relation à l'incertitude, non plus problème, mais outil voire force de l'artiste interpellé et inquiété par le réel.

3 La dimension visuelle des travaux de Banu Cennetoglu - comme nous l'indique l'artiste au cours de l'entretien qu'elle nous a accordé - étant essentielle, nous invitons le lecteur à consulter également son site personnel (http://www.banucennetoglu.com) ${ }^{1}$.

A propos de False Witness

4 Banu CENNETOGLU (B.C.) : J'ai décidé de faire ce projet après avoir visité un centre d'enregistrement de demandeurs d'asile (étape antérieure donc à l'attribution du statut 
de réfugié) appelé "Ter Apel ", en Hollande. Soumis à une procédure d'examen de 48 heures (16 heures pendant trois jours), les demandeurs d'asile ne peuvent quitter le bâtiment pendant ce temps. Ce centre, le dernier à s'être ouvert en Hollande (il y en a cinq) est assez particulier du point de vue architectural aussi bien à l'intérieur qu'à l'extérieur. Les demandeurs d'asile doivent suivre un parcours "en accord» ou parallèle à la structure du bâtiment. En voici quelques exemples. Lors de leur entrée, au tout début de la procédure, les demandeurs d'asile doivent franchir une porte bleue car le bleu symbolise "le froid et l'Est». S'ils sont autorisés à séjourner pendant une certaine période en Hollande en vue d'obtenir le statut de réfugié, ils quittent le centre en passant par le côté orange et se rendent dans un camps où ils seront logés dans des caravanes (autrefois ce séjour pouvait durer six ans ; il est désormais d'au maximum six mois, laps de temps pendant lequel une décision doit être prise). Les pièces où ont lieu les entretiens sont également divisées en couleurs. La personne qui conduit l'entretien est assise dans la partie blanche («neutre»), le demandeur d'asile est assis dans la partie colorée (la couleur est censée symboliser les bagages, le passage).

que ces explications relatives aux «symboles » des couleurs ne sont pas de mon cru: elles m'ont été fournies par la personne responsable des relations publiques du centre.

6 «False Witness » se présente comme un livre mais non comme un livre reportage sur Ter Apel. Je suis intéressée par le potentiel de la photographie et la possibilité de l'invisible qui peut naître du visible. Dans cette optique, j'ai voulu trouver un moyen de combiner la documentation du centre et d'autres images, le tout étant amené à devenir autre chose. Une sorte de troisième situation. Plutôt que de crier «ça ne va pas ", ce projet entend ouvrir une discussion. Le texte situé au début du «livre acte » agit comme faux index. Ce sont des fragments du «Corpus Base Data » pour le mot mesure. Un «Corpus Base Data » est une compilation d'informations que les gens qui font des recherches avant de produire un dictionnaire utilisent. Ils collectent à partir des diverses sources des exemples pour montrer les différents comportements d'un mot. J'ai donc utilisé ces fragments comme une source et je les ai terminés comme je l'ai voulu. A la fin, ils existent en tant que phrases indépendantes et aussi - à cause de mon interférence - comme un seul texte. Le texte et les photographies ont une structure parallèle mais indépendante l'une de l'autre. Au demeurant, il me semble important de garder l'ensemble comme tel et c'est ce qui est exposé.

$7 \quad$ C\&C : Le livre a-t-il d'autres supports de diffusion que l'exposition? Peut-il être diffusé autrement qu'en exposition? Le souhaitez-vous?

B.C. : Oui, en effet. Lorsque j'ai conçu False Witness comme un livre, je voulais voir si un livre pouvait fonctionner comme une exposition, un espace de projet en tant que tel. C'est également pour cela que lorsque je le montre je souhaite garder rassemblé le contenu du livre et ne pas exposer simplement les photos. En dehors des expositions, le livre est diffusé dans des librairies.

$9 \quad$ C\&C : En fonction de ce que vous nous avez communiqué au sujet des particularités architecturales et des dispositifs du centre Ter Apel: peut-on dire qu'il y a un certain parallèle entre la visite de l'exposition et la procédure de demande d'asile telle qu'elle se donne dans ce centre?

10 B.C. : Non, pas directement. Mais il y a des raisons conceptuelles et formelles à la construction d'un corridor comme traduction spatiale du livre. Trois corridors consécutifs donnent un espace construit de $12 \mathrm{~m}$ x $90 \mathrm{~cm}$ et le livre entier est imprimé 
en trois bandes correspondant à chaque corridor. Du fait de l'étroitesse de l'espace dans lequel on se déplace, le visiteur ne peut pas scanner les images d'un seul coup d'œil. En entrant dans l'espace, on entre "physiquement " dans le livre et il est nécessaire de traverser tout l'espace pour pouvoir le terminer.

11 C\&C : Avez-vous eu des contacts avec des demandeurs d'asile lors de l'élaboration de cette oeuvre?

B.C. : Non, je n'ai eu aucun contact avec des demandeurs d'asile pendant l'élaboration de ce projet. J'en ai décidé ainsi. Je voulais travailler surtout sur l'espace comme illustration de cet état d'esprit " particulier ». Et, du point de vue éthique, je trouvais cela incorrect de travailler avec des demandeurs d'asile dont on connait la situation pour le moins délicate, d'extrême fragilité, soumis qu'ils sont à des problèmes auxquels je ne pouvais apporter aucune solution concrète.

13 En réalité, pendant mon projet, Ter Apel était un centre d'enregistrement pour demandeurs d'asile. Depuis juin 2004, il est devenu un centre de déportation ${ }^{2}$ pour quelques 26000 demandeurs d'asile dont les demandes ont été refusées et que les PaysBas souhaitent « déporter » entre 2004 et 2007.

Le gouvernement a décidé de poursuivre les déportations au cours des trois prochaines années des demandeurs d'asile arrivés au pays avant avril 2001.

C\&C : Avez-vous pris (pu prendre, voulu prendre) des photos au centre Ter Apel? Quelles sont les autres images utilisées, leurs sources? Comment ces deux supports images dialoguent-ils?

B.C.: Oui, j'ai pris des photos. Lorsque j'ai envoyé ma proposition au Centre pour obtenir la permission pour les visites je leur fis savoir que c'était pour une « recherche ». L'une des principales raisons de mon intérêt pour Ter Apel est que le processus impliquant le demandeur d'asile est parallèle à la structure architecturale du bâtiment. Je me suis donc engagée dans ma proposition à ne déranger aucun demandeur d'asile en le photographiant. De ce fait, je n'ai pris que des photos du bâtiment et des structures architecturales. Plus tard, dans le livre, je me suis servi de ces photographies pour constituer le scénario principal.

17 Je l'appelle donc « scénario principal » car il y a des photographies provenant d'autres histoires.

Ces autres images sont issues du monde entier. Ce sont des images collectées pendant plusieurs années et en des endroits extrêmement divers (crèches gouvernementales à Batumi; logements sociaux à Quito; environs de Chamarande en France). Elles ont leurs propres histoires mais je voulais qu'elles interfèrent dans ce contexte avec mon scénario principal et que cela créé d'autres possibilités. La sélection et l'ordonnancement de ces photographies ne tiennent pas au hasard. C'est même loin d'être le cas. Je crois avoir passablement manipulé leur interaction potentielle. Le livre n'illustre pas un sujet mais utilise un fait documenté comme source. Ensuite, les associations entre les images deviennent cruciales.

Il s'agit plus d'une discussion que d'une déclaration.

20 C\&C : Concernant le Corpus Base Data et le mot «mesure »: pourquoi la notion de mesure?

21 B.C. : Je travaille beaucoup sur la notion de distance, en particulier sur la manière dont nous percevons et traitons l'information. Je pense que dès lors que nous recevons 
l'information, nous créons un espace mental en fonction de son « poids » qui génère un moyen possible de la traiter. J'ai choisi le mot «mesure " à cause des possibilités d'associations riches, variées et critiques qu'il peut offrir. semaine. Au cours de la même période il y aura également une diffusion sauvage via le collage de listes imprimées. La seconde partie du projet inclue un centre de documentation. SKOR est une initiative sans but lucratif lancée par des artistes. Elle bénéficie d'un petit bâtiment localisé sur les abords d'un quartier très fréquenté de la ville. Ce lieu comprendra des documents, des ouvrages, des textes imprimés et des vidéos traitant de ce sujet. Nous travaillons actuellement à la collaboration avec un journal local pour la diffusion de la liste pendant cette même semaine. La troisième partie du projet comprend des projections, des discussions et des présentations à l'université d'Amsterdam. 

Möbius. Depuis un certain temps mes lectures et mes réflexions me mènent également à la question du pouvoir de la potentialité. D'Aristote à Agamben, cette discussion est continue. Mais il est très important de voir le point de vue d'un professeur de relations internationales qui peut faire confiance à l'incertitude en traitant des nouveaux systèmes de sécurité au sein de l'UE. C'est un point d'inspiration et d'influence.

Bien que nous soyons sans cesse exposés à l'information, nous sommes plus indifférents que jamais. En tant qu'artiste je ressens le devoir de créer une proximité entre l'information fournie par cette liste et le public.

Je considère que la liste doit être rendue publique et aussi visible que possible. Bien que l'information soit déjà disponible sur le site Internet de l'organisation United (www.united.non-profit.nl) ${ }^{4}$, je crois en l'extrême nécessité de sa diffusion et à l'effet de sa confrontation. En la plaçant dans une structure culturelle-commerciale au sein de l'espace urbain, l'assurance de la confrontation avec un public grandit: si le public rate la liste le matin, il devra passer devant sur le chemin du retour.

Pour moi «The List » - le projet - n'est ni une oeuvre d'art ni un acte politique. Je pense que par le biais des frontières indéfinies de l'art ainsi que par l'exploitation de ma position d'artiste, il est possible de rendre visible les préoccupations inhérentes au contenu de la liste ainsi que de tester les limites de l'information publique. L'art n'est pas CNN. Il peut, par un médium, offrir ou imposer des informations inattendues à un moment inattendu. Ce qui est justement son pouvoir.

Les frontières entre un art socialement engagé et son inverse autonome sont en cours de discussion, tout comme les frontières entre l'interne et l'externe, du fait de la limite atteinte de notre imagination politique. Je suis tout à fait consciente de la fragilité de ce matériel ainsi que de l'indifférence chronique de la société dans laquelle je souhaite la rendre visible.

4 C\&C : Comment s'est fait le contact avec l'université d'Amsterdam? Quel accueil a été donné à votre travail ? Y a-t-il eu des difficultés?

B.C. : Via mon partenaire dans ce projet, Huib H. van der Werf qui était autrefois étudiant à l'université d'Amsterdam. L'université s'est intéressée à notre projet et a souhaité collaborer. Nous n'avons donc rencontré aucune difficulté de ce point de vue.

C\&C : Le projet comprend donc également un dialogue (dans le cadre d'un colloque) avec des artistes et des universitaires : comment s'est fait le choix des participants? Comment l'idée d'un tel colloque a-t-elle été accueillie? Par les universitaires? Par les artistes?

B.C. : En dehors de l'existence de la liste, l'ouvrage de Carlo Ginzburg, Wooden Eyes and 9 Perspectives on Distances, (« des yeux de bois et neuf perspectives des distances ») a été un déclencheur majeur de ce projet. A partir de ce livre, ma question était « ce qui est loin des yeux est-il loin de l'esprit?».

8 Puis, j'ai lu le texte de Didier Bigo sur les systèmes de sécurité et je me suis intéressée de près à la question de l'incertitude comme possibilité et à la métaphore du ruban de En mars 2004, j'ai participé à la $3^{\mathrm{e}}$ biennale de Berlin et j'y ai rencontré les travaux de Hito Steyerl March, auteur et réalisatrice qui se sert du ruban de Möbius comme métaphore pour analyser la nouvelle structure de l'Europe. Ensuite, ce fut le film merveilleux de Zelimir Zilnik : Kennedy goes home. Un film très intense et intelligent sur un sujet extrême. Zilnik est pour moi le meilleur exemple du questionnement par le film. 

long de ce que l'on a appelé plus tard les frontières Schengen. Il a pris des photos. Son approche directe m'intéresse énormément tout comme la question de savoir si une information directe peut mener à une action directe. J'ai fait attention à choisir des personnes d'origines disciplinaires différentes ayant un engagement sincère sur cette question, et j'ai réussi à les rassembler en deux ans.

Art et politique : exploiter l'incertitude

41 C\&C : Comment pourriez-vous nous présenter ce qu'est votre travail tous les jours? Quels en sont les piliers?

B.C. : Il est peut être mieux de commencer en vous donnant quelques informations sur mon parcours personnel. Après avoir étudié pendant quatre ans la psychologie à Istanbul (d'où je suis originaire), j'ai déménagé à Paris et j'ai étudié la photographie à Speos. Ces études étant terminées, je me suis installée à New York où tout en travaillant comme photographe professionnelle je développais mes projets personnels axés sur un questionnement sur la photographie et l'information (en particulier sur la production et la distribution de l'information). Finalement, ces questions ont pris une telle ampleur quantitative et qualitative que j'ai choisi d'abandonner mon activité professionnelle pour me consacrer au maximum à mon travail personnel. C'est très précisément à cette période que l'Académie Rijksakademie à Amsterdam m'a octroyée une bourse de deux ans.

La bourse était destinée à un programme de recherche de post-grade sur l'art contemporain. C'est là que j'ai commencé à concevoir «False Witness» et progressivement « The List ».

Je travaille essentiellement à partir de la photographie : ce qui m'intéresse c'est de photographier des espaces dont les identités sont transitoires et instables. Par ce processus, je vise à questionner le potentiel de ces espaces/lieux et le pouvoir de leur condition incertaine.

45 Je ne me promène pas avec mon appareil photo à longueur de temps. Je travaille très souvent dans mon studio en me laissant de longues périodes de recherche. Dans la mesure où mes projets impliquent de la distance et des voyages, je ne suis pas très productive sur des périodes courtes. Je préfère travailler longtemps sur un projet et me concentrer sur ses différentes « couches ». Par couches, j'entends les différentes parties qui, ensemble, constituent le tout. Elles peuvent avoir des épaisseurs et des poids différents, soit des niveaux d'intensité et d'importance différents. Les couches sont importantes car elles indiquent la profondeur d'un travail. Même si chacune d'entre elles n'est pas visible explicitement, le fait qu'elles aient toutes été considérées affecte la perception et la lecture de l'œuvre finale.

Quelle est mon approche d'un espace et de son histoire, et ensuite la relation entre la photographie et son contexte d'origine? Lorsqu'une image est présentée, elle offre de multiples possibilités d'interprétation.

Je pars du terrain et en le photographiant, je construis son histoire. Ce terrain particulier est situé dans ce pays de sorte que les couches d'information se rejoignent en fonction de l'état économique, social et politique de ce même pays. Je m'intéresse donc à ce terrain, isolé, mais aussi au contexte élargi de sa localisation. 

propres limites. J'ai entendu un certain nombre de questions ces derniers temps sur la mission de l'art : l'art devrait-il avoir une mission? Etes vous artiste ou anthropologue? peut être utilisé de différentes manières. Pour reprendre les termes de Didier Bigo «le fait de ne pas savoir sur quelle face du ruban on se trouve n'est pas un danger mais plutôt une opportunité ». ni comme un acte politique car pour moi il s'agit de le diffuser et de pouvoir soulever des questions. Je suis une médiatrice, pas une créatrice dans ce projet. J'aime utiliser le système dans lequel je fonctionne, diffuser cette information du fait de l'urgence de sa nécessité. Je ne le considère pas comme un acte politique car j'utilise un moyen indirect de communication. Je cours le risque de ne pas être vue ou entendue. "The List» 
pourrait être un exemple de ce qu'Agamben nomme "moyens sans fins", ceci dans le contexte du cinéma qui, selon Agamben, a son centre dans le geste et non l'image et appartient donc essentiellement au monde de l'éthique et de la politique et non plus simplement à celui de l'esthétique.

61 "Ce qui caractérise le geste, c'est qu'il ne soit plus question en lui ni de produire ni d'agir, mais d'assumer et de supporter. Autrement dit, le geste ouvre la sphère de l'ethos comme sphère la plus propre de l'homme. Mais comment une action est-elle assumée et supportée ? Comment une res devient-elle res gesta; et un simple fait, un évènement ? (...) si le faire est un moyen en vue d'une fin et l'agir une fin sans moyens, le geste rompt la fausse alternative entre fins et moyens qui paralyse la morale, et présente des moyens qui se soustraient comme tels au règne des moyens sans pour autant devenir des fins $»^{5}$.

C\&C : La durée de vie de vos œuvres?

B.C.: Mes travaux ont deux vies. La première est celle de leur conception et production. L'autre est celle du travail terminé lorsque les personnes commencent à s'y intéresser. Et il y a une grande différence temporelle entre ces deux vies. Par exemple, j'ai commencé un projet sur l'ex-Yougoslavie en 2002, je l'ai terminé en mars 2004. La première exposition a eu lieu en mai 2004. Après cette première exposition, rien ne s'est passé avant septembre 2005. Puis, j'ai montré ce même travail quatre fois au cours des quatre derniers mois. C'est une situation étrange, les gens ont besoin d'assurances ou de références avant d'inviter le travail pour des expositions, c'est-à-dire qu'un projet nouveau a toujours du mal à démarrer.

C\&C : Dans la présentation de vos œuvres, le visuel semble s'imposer sur l'écrit (même si l'un et l'autre sont sollicités). Peut-on dire que pour vous il y a une primauté du visuel? Si c'est le cas, pourquoi? Ou, que permet le visuel? Ces questions nous intéressent en particulier autour de certaines considérations que vous avez eu au moment de présenter vos œuvres : la possibilité de l'invisible qui peut naître du visible ; la volonté de diffuser, d'établir une médiation (comme vous venez de le dire) mais aussi l'envie de communiquer et même la nécessité d'instaurer un dialogue. Pourriez-vous nous en dire plus?

65 B.C. : Oui. Il y a clairement une primauté du visuel dans mon travail. Je suis d'abord une artiste du visuel et je considère et je souhaite que les photographies génèrent la possibilité d'associations et de discussions.

Lorsque je dis que l'invisibilité peut naître du visible, je fais référence à l'espace imprévisible où les oeuvres peuvent amener le public par le biais du matériel existant. La photographie a une nature conflictuelle. Tandis qu'elle montre ce qui est là, elle ne peut changer la situation de ce qui est pris en photo. C'est pour cela que je m'intéresse à la façon de construire une narration susceptible d'aller au-delà d'une illustration ou d'une éducation. Il ne s'agit donc ni simplement d'illustrer à la manière du reporter ni de donner des leçons. Cette réflexion est liée à la notion de dialogue que je développe par ailleurs.

67 Je considère que c'est le seul moyen d'avoir un dialogue au lieu d'un monologue. En général, lorsque les personnes regardent " False Witness », soit cela ne les intéresse pas du tout et elles abandonnent le livre, soit cela les intéresse et le dialogue commence.

C\&C : Vous avez justement évoqué le thème de l'indifférence. Qu'est-ce que l'indifférence ? Pour nous, sociologues, c'est l'impossible interlocuteur, et en même 
temps, un puissant moteur d'action : beaucoup d'expériences politiques, et notamment certaines expériences "novatrices" en politique ont pour motivation première, immédiate, la nécessité de rompre cette indifférence. Comment intervient l'« Indifférence » dans vos œuvres ?

B.C.: L'indifférence est normale et représente un mécanisme d'autodéfense très efficace. L'indifférence collective est dangereuse, en particulier dans le monde dans lequel nous vivons. Nombreux sont ceux qui ne peuvent le supporter que par l'indifférence.

70 J'ai fortement conscience de cela mais je n'agis pas sur cela. Le seul moyen de changer cette situation est de l'ignorer. Je ne pense pas que nous puissions l'arrêter mais nous ne sommes pas non plus obligés de l'arrêter. Nous devons continuer, même si à première vue cela ne semble faire aucune différence. Mais je suis persuadée que cela en fera... tôt ou tard. Avez-vous vu le film TAKE de Naomi Klein et Avi Lewis? Un documentaire sur le comment les travailleurs en Argentine reprennent une usine après la crise économique de 2001. Un exemple merveilleux de comment faire la différence !

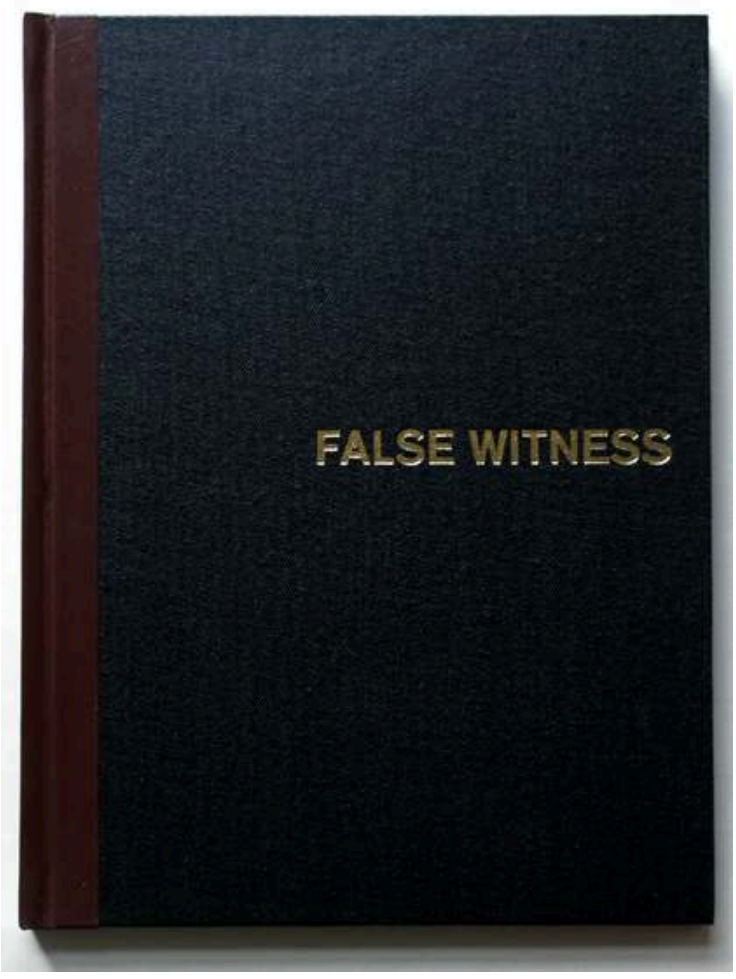

(c) Banu Cennetoglu 


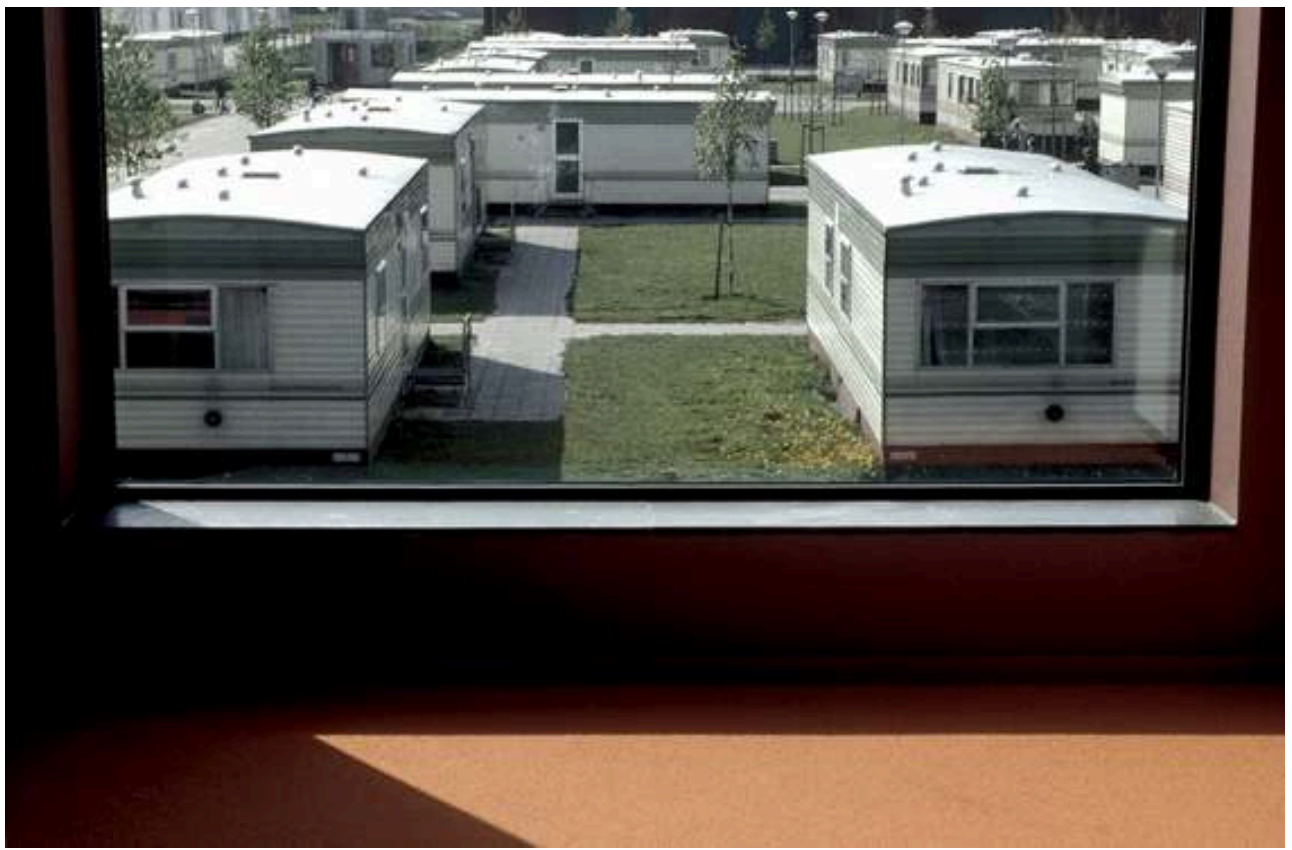

(C) Banu Cennetoglu

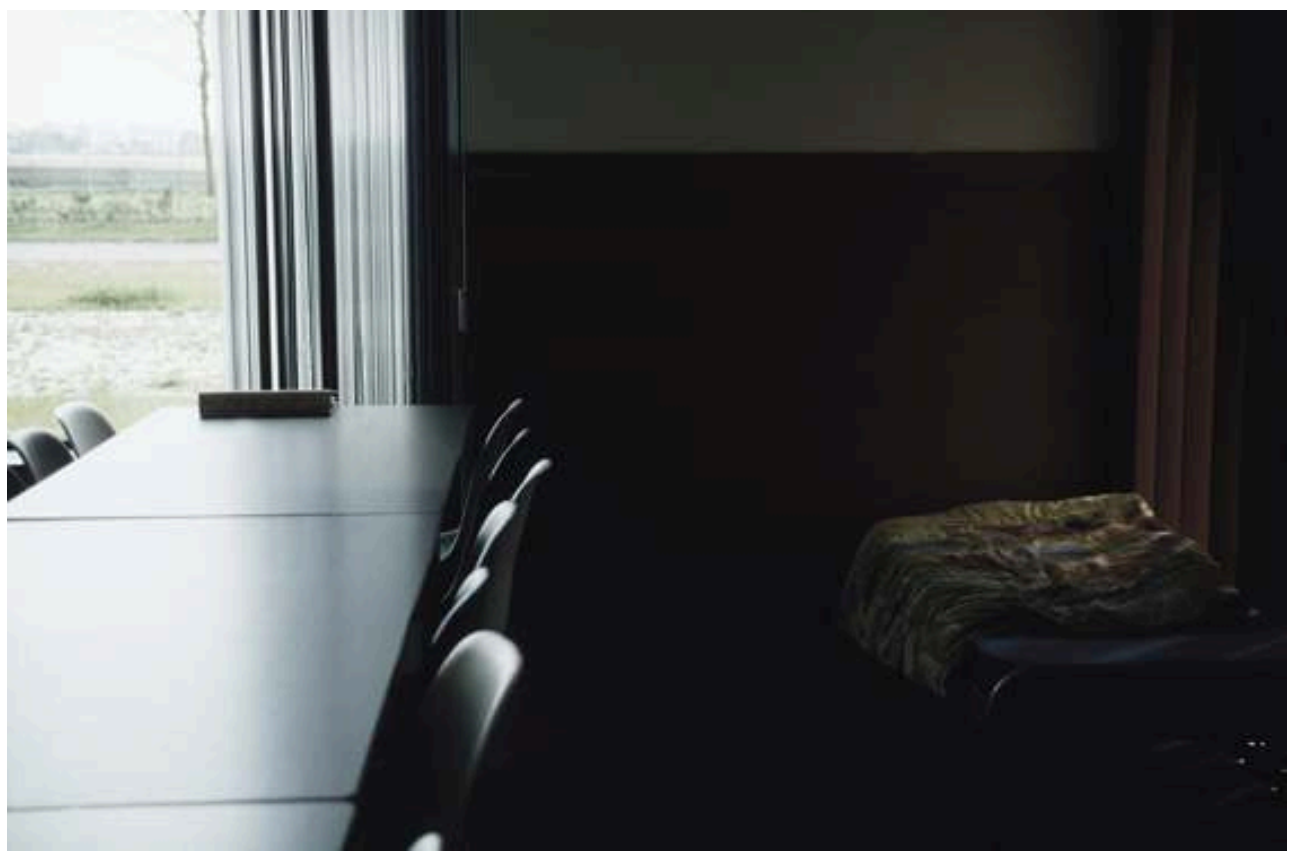

(c) Banu Cennetoglu 


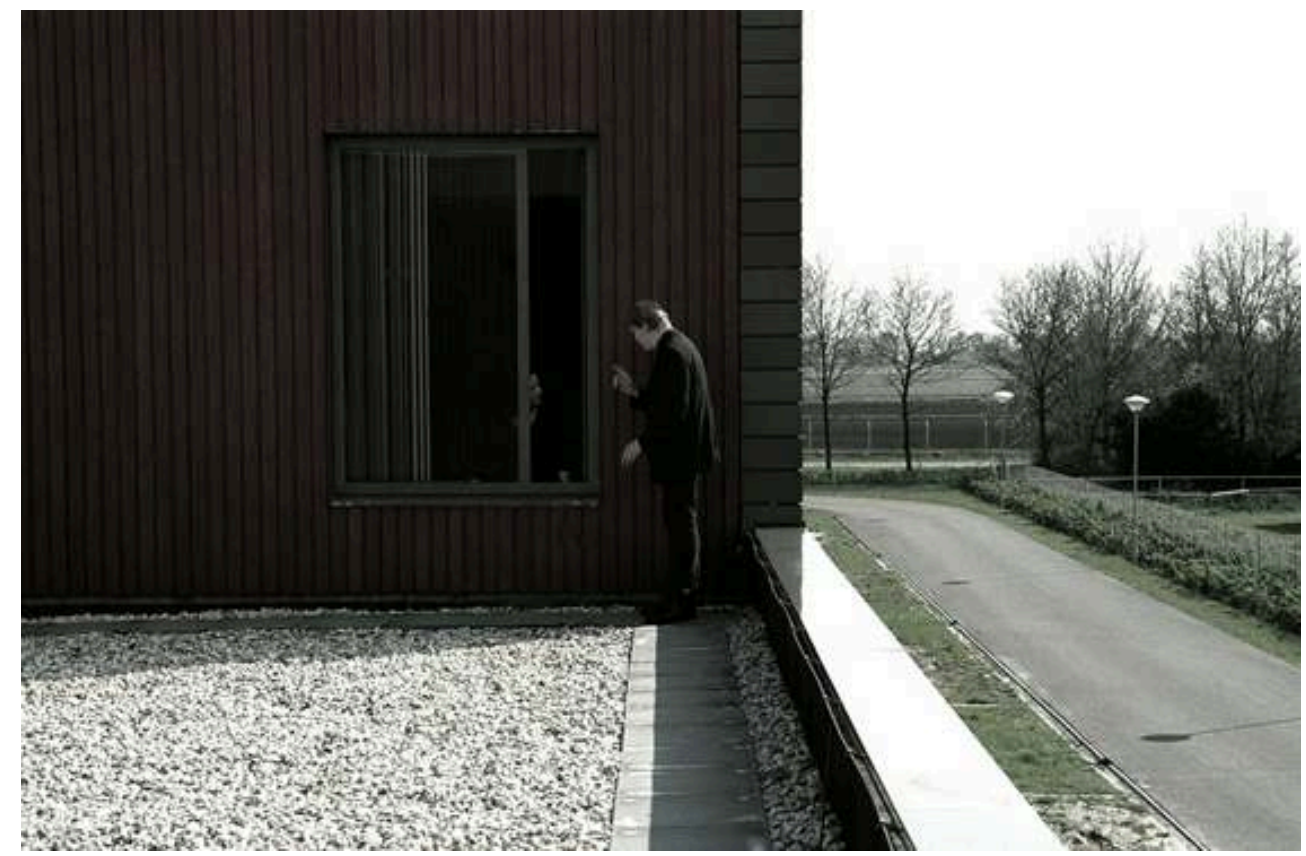

(c) Banu Cennetoglu

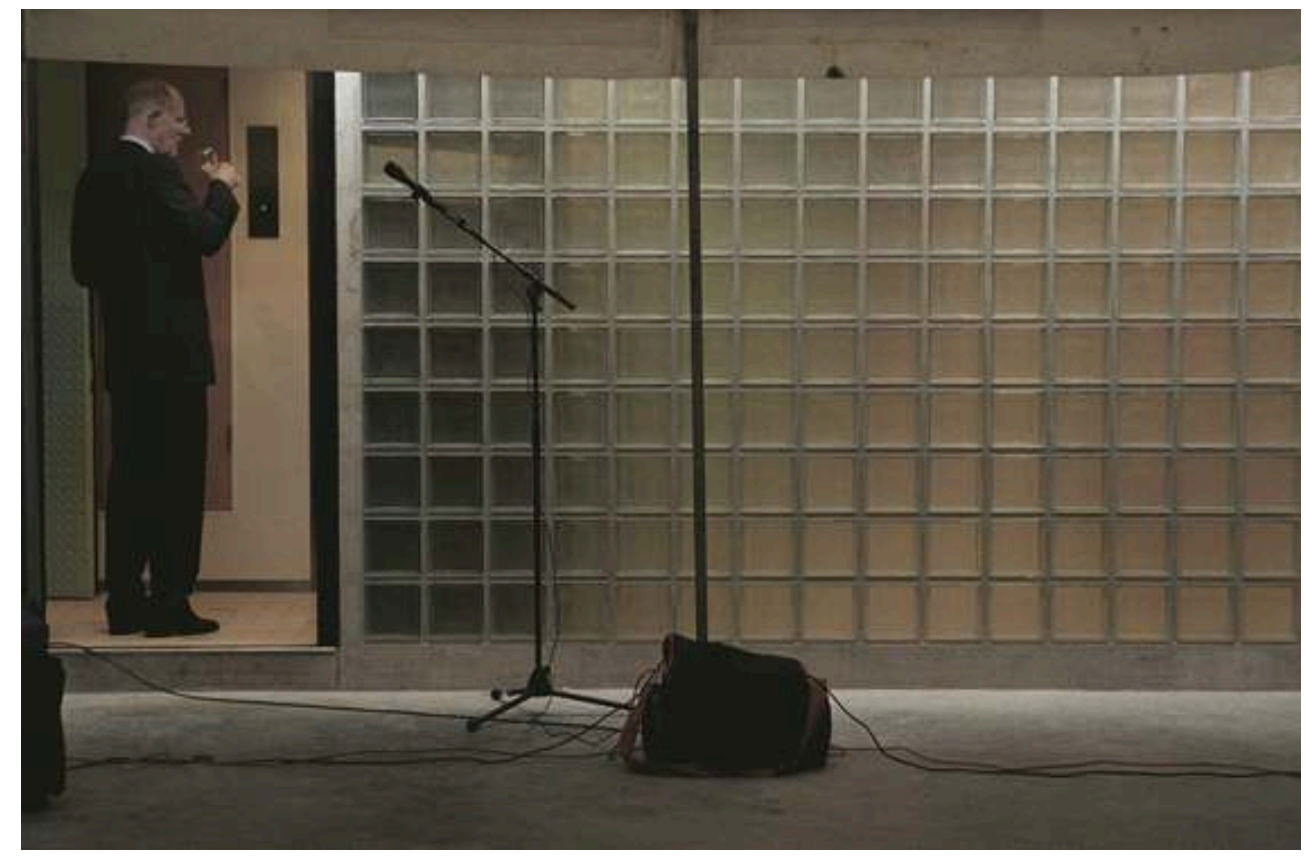

(c) Banu Cennetoglu

Toutes ces photographies sont extraites de l'ouvrage False Witness. 


\section{NOTES}

1.. Ce dossier prend appui sur les informations disponibles sur ce site et sur un dialogue direct avec l'artiste pendant les mois d'août et de septembre 2005. Le dossier est également disponible en anglais sur www.conflits.org. Un entretien à distance a par ailleurs été réalisé en octobre 2005.

2.. Le terme « déportation » est celui employé officiellement pour désigner les centres par lesquels passent les étrangers avant d'être expulsés du pays.

3.. Il s'agit ici d'extraits de l'ouvrage False Witness : « 378858 M. Mauvais utilise de faux poids et mesures et trompe ses créanciers par une fausse banqueroute. 110487 tous doivent faire preuve de bonne volonté et trouver les mesures d'urgence appropriées. 331431 aux Etats-Unis, les poids et les mesures ont été standardisés par le bureau national. 037783 je suis différent...je suppose que ne me suis jamais contenté de demimesures, je regrette que ta vie soit si pesante ». Traduction de la rédaction.

4.. United est un réseau européen contre le nationalisme, le racisme et le fascisme qui assiste les migrants et les réfugiés. Il est localisé à Amsterdam.

5.. Agamben G., Moyens sans fins (notes sur la politique), Payot et rivages, 1995, p. 68.

INDEX

Mots-clés : art, entretien, regards sur l'entre-deux

\section{AUTEURS}

\section{ANTONIA GARCIA CASTRO}

Antonia Garcia Castro est docteur en sociologie, co-rédactrice en chef de la revue Cultures \& Conflits.

\section{MIRIAM PERIER}

Miriam Perier est titulaire d'un DEA de sociologie politique (Université Paris-I) et chargée de recherches au centre d'études sur les conflits. 